\title{
CRISIS ECONÓMICA MUNDIAL DEL 2008 Y SU IMPACTO EN LA EVOLUCIÓN DE LA ECONOMÍA PERUANA
}

\author{
GLOBAL ECONOMIC CRISIS OF 2008 AND ITS IMPACT ON THE EVOLUTION OF \\ THE PERUVIAN ECONOMY
}

Bernardo Javier Sánchez Barraza Universidad Nacional Mayor de San Marcos Lima, Perú

ORCID: https://orcid.org/0000-0001-6755-388X Correo electrónico: bsanchezb@unmsm.edu.pe

\section{RESUMEN}

Objetivo: Determinar el impacto de la crisis de la economía mundial del año 2008 en la evolución y proceso de la economía peruana durante el periodo 2008-2014. Método: Investigación no experimental, transversal y retrospectiva; el alcance descriptivo, correlacional, explicativo; y el enfoque cuantitativo, con base en la medición, la evaluación numérica y el análisis estadístico. Resultados: Se descarta que la relación entre el crecimiento del PBI para el periodo 2008-2014 y la crisis investigada sea nula, y según los resultados del modelo ARIMA se encuentra convicción y evidencia de una relación negativa entre este período y la economía del Perú. Asimismo, es importante subrayar que, en la ausencia de una significancia individual, la correspondencia no ha sido necesariamente cercana o directa, sino que se mostró de forma tardía en la economía peruana. Conclusiones: Los acontecimientos económicos ocasionados a una escala macroeconómica impactan a la economía global, y como el Perú es parte de ella, siempre se va a afectar positiva o negativamente; no obstante, el nivel de impacto dependerá de los vínculos y acuerdos que pueda sostener.

Palabras clave: Crisis económica; economía; evolución; impacto; mundial.

\begin{abstract}
Objective: Determine the impact of the global economy crisis in 2008 on the evolution and process of the economy in Peru during the period 2008-2014. Method: Non-experimental, transversal and retrospective research; the scope is descriptive, correlate, explanatory; quantitative approach, based on measurement, numerical assessment and statistical analysis. Results: It is ruled out that the relationship between GDP growth for the period 2008-2014 and the crisis investigated is null, and according to the results of ARIMA model is found conviction and evidence of a negative relationship between this period and the economy of Peru. Likewise, it is important to underline that in the absence of individual significance, the correspondence has not necessarily been close or direct, but was belatedly shown in the Peruvian economy. Conclusions: Economic events caused a macroeconomic scale impact the global economy, and as Peru is part of it, it will always be positively or negatively affected; however, the impact level will depend on the links and agreements that it can sustain.
\end{abstract}

Keywords: Economic crisis; economy; evolution; impact; global.

(c) Los autores. Este artículo es publicado por la Revista Quipukamayoc, Universidad Nacional Mayor de San Marcos. Este es un artículo de acceso abierto, distribuido bajo los términos de la Licencia Creative Commons Atribución-NoComercial-Compartirlgual 4.0 Internacional.(http://creativecommons.org/licenses/by-nc-sa/4.0/), que permite el uso no comercial, distribución y reproducción en cualquier medio, siempre que la obra original sea debidamente citadas. 


\section{INTRODUCCIÓN}

El estudio analiza el impacto de la crisis y del desequilibrio económico a escala mundial acaecido en el 2008 en la economía peruana, específicamente, en el Producto Bruto Interno en relación con las principales variables económicas de países del Grupo de los Siete - G7 (EE.UU, Alemania, Canadá, Francia, Italia, Japón, Reino Unido).

Para la comprensión integral de la temática, se exponen los fundamentos y aspectos teóricos referidos a la crisis económica y financiera, y al Producto Bruto Interno (PBI). Mankiw (2014) afirma que "para medir el crecimiento económico, los economistas emplean datos sobre el Producto Bruto Interno, que mide la renta total de todos los miembros de la economía” (p. 309).

Akerlof y Shiller (2014) sostienen que las crisis económicas, como la crisis financiera de 2008, se han producido, principalmente, debido al cambio de patrones de pensamiento de la sociedad, lo cual va en contra de las ideas habituales de la economía. Desde tal perspectiva, se puede afirmar que la crisis del año 2008 se debió al surgimiento de patrones de pensamiento, de manera específica, a variaciones de la confianza, las cuales están asociadas a las percepciones acerca de la naturaleza de la economía. El exceso de confianza, sea individual o colectiva, ocasiona que las personas rechacen una información, incluso aunque la procesen, porque con mucha frecuencia se sienten confiadas en que la decisión que están tomando es la mejor.

Por su parte, Herrera (2003) afirma que las crisis financieras, como la bancarrota de una institución bancaria, la morosidad de la amortización de la deudas o préstamos externos, una devaluación monetaria, o una conjunción de todas o varias de ellas, que lleva irremediablemente a la disminución de la actividad económica y en los niveles de ingreso.

En efecto, cualquier crisis financiera como las precisadas conllevan a la caída de los niveles de producción, con lo cual se evidencia el nexo que existe entre el sector financiero y el sector real de la economía. Justamente, debido a esta interconexión que existe entre ambos sectores, las políticas públicas en esta materia deben tener por objeto promover la estabilidad de los mercados financieros a efectos de mantener la estabilidad financiera y, a su vez, reforzar la estabilidad macroeconómica y el crecimiento.

Krugman (2009), al referirse a la crisis, considera que la subida de precios y valores de las viviendas llegó a un nivel que adquirir una casa ya no estaba dentro de las posibilidades de compra de un gran número de personas en EE.UU, a pesar de que ya no se pedía la primera cuota de crédito o incluso se brindaba financiamiento con tasas bajas; la venta de viviendas comenzó a disminuir.

Lo expresado en el párrafo precedente permite comprender que el comercio de casas y acciones tienen comportamientos diferentes. Las transacciones que se realizan en el sector inmobiliario se mueven a un ritmo menor que el comercio de acciones. También se debe tomar en cuenta que la vivienda es un aspecto más importante que la Bolsa del Mercado de Valores, en especial para las personas y familias de nivel socioeconómico medio, cuyas casas son usualmente su activo o patrimonio principal. Es así que al inicio de la década previa, las tasas de interés se encontraban relativamente bajas, las cuales favorecían a la adquisición de viviendas diversas. Usualmente los norteamericanos compran sus propiedades con dinero financiado por instituciones crediticias. En cierta medida esto se fomentó por el exceso individual de las personas y familias que observaron cómo los precios de las viviendas y propiedades aumentaban ostensiblemente y decidieron comprar en este mercado despreocupados en cómo realizarían los pagos futuros. A los demandantes de créditos se les brindó los préstamos que necesitaban con una cuota inicial mínima o cero, y con cuotas mensuales que sobrepasaban su capacidad de amortización o pago de deuda. A varios de estos préstamos dubitativos, pero no a todos, se les denominó subprime.

Para Stiglitz (2013) la crisis de las hipotecas fue originada por la sobreliquidez en los mercados financieros y además considera que uno de los grandes responsables fue el Banco Central. En sus palabras:

La crisis de las hipotecas subprime (hipotecas de alto riesgo), que conllevó una crisis más amplia en los mercados de crédito, fue originada en cierto modo por una sobreliquidez en los mercados de financiamiento y capitales internacionales, y también por los defectos en los bancos centrales en Estados Unidos $y$ en varios países de economías fuertes y que en el momento de proceder para frenar la liquidez y menguar el incremento de las quimeras especulativas en el mercado del sector vivienda y otros valores de activos similares, sus decisiones no tuvieron buenos resultados. (p. 71)

Las hipotecas subprime hacen referencia a las hipotecas de alto riesgo, se caracterizaban por ser de tipo específico, ya que eran empleadas mayormente para la compra de propiedades y viviendas, direccionadas a clientes de bajo poder adquisitivo, y por resultado, con un nivel o escala de riesgo de morosidad superior al promedio del remanente de créditos o financiamientos. Otro aspecto importante de estos créditos hipotecarios era la prominente tasa de interés, en comparación con los préstamos 
individuales o personales, a pesar de que en los primeros años se brindaban tasas promocionalmente atractivas.

Sunstein y Thaler (2017) dan una explicación a la crisis financiera desde el punto de vista conductual, afirman que:

Una causa clave del desastre de las hipotecas subprime es que muchos prestatarios no comprendian las condiciones de sus préstamos. Incluso los que intentaban leer las páginas en letras pequeñas se sentían flaquear, especialmente cuando su agente hipotecario les aseguraba que habían conseguido unos términos excelentes. (p. 29)

En definitiva, no se tienen antecedentes previos directos sobre las variables investigadas (crisis económica mundial de 2008 y Producto Bruto Interno peruano), debido a que las investigaciones existentes tratan fundamentalmente el impacto de la crisis en la economía mundial del año 1998 y en el sistema financiero del Perú.

Dentro de este contexto, el objetivo de la investigación es determinar el impacto de la crisis y el desequilibrio económico a escala mundial del 2008 en la economía peruana durante el periodo 2008-2014. Desde tal perspectiva, la hipótesis que responde a la pregunta es: La crisis económica mundial de 2008 impactó moderadamente en la economía peruana durante el periodo 2008-2014.

\section{MATERIALES Y MÉTODOS}

El diseño de investigación fue no experimental porque se analizaron los fenómenos tal como ocurrieron, sin intervenir en su desarrollo. El alcance de la investigación fue descriptiva, correlacional y explicativa. Descriptiva, porque se analizó la evolución de la economía peruana, así como las principales consecuencias de la crisis financiera de 2008. Correlacional, porque se relacionaron las variables de estudio: evolución del PBI peruano respecto de las principales variables macroeconómicas de los países del G7. Explicativa, ya que centró en explicar e interpretar por qué la crisis y el desequilibrio económico a escala mundial del 2008 marcó e impactó en el desarrollo del Producto Bruto Interno peruano durante el periodo investigado. El enfoque fue cuantitativo, puesto que es probatorio por medio de data estadística, y las unidades analizadas fueron los datos del Producto Bruto Interno peruano y los datos de las principales variables de las economías de los países del G7.

El análisis e interpretación de la información se realizó mediante la revisión de la información publicada por el Fondo Monetario Internacional (FMI), la Organización para la Cooperación y el Desarrollo Económicos (OCDE), el Banco Central de Reserva del Perú (BCRP). A partir de los datos obtenidos de las variables de interés, se empleó el análisis descriptivo para observar el impacto de la crisis de 2008 en la economía peruana, y a través de exámenes estadísticos (modelos regresivos) para establecer el impacto entre las variables de interés. En suma:

$$
\begin{aligned}
& \mathrm{X}=\text { Crisis económica mundial de } 2008 \\
& \mathrm{Y}=\text { Producto Bruto Interno peruano }
\end{aligned}
$$

$$
\mathrm{Y}=\mathrm{f}(\mathrm{X})
$$

$\mathrm{Y}=$ Var. efecto (Producto Bruto Interno peruano)

$\mathrm{X}=$ Var. causa (Crisis económica mundial del 2008)

Se optó por una muestra no probabilística o dirigida denominada muestra de casos-tipo, cuyos objetivos son la riqueza, profundidad y calidad de la información y no la cantidad o estandarización de esta.

De esta forma, se utilizaron dos modelos regresivos para estimar el impacto entre las variables (X e Y):

En primer lugar, se hizo uso del modelo Autorregresivo Integrado de Medias Móviles (ARIMA) para analizar las series de tiempo trimestrales de las variables dependientes e independientes. En este caso, se utilizó únicamente la parte Autorregresiva (AR) por el rol que cumplen los rezagos para explicar la misma variable. Como herramienta regresiva se hizo uso del software Eviews 8.0. Se considera autorregresivo porque la variable interna o endógena de un período o lapso t es comprendida en base a las observaciones de ella misma en concordancia con períodos previos y adicionándole, como en los modelos de estructura, el llamado término de error.

En tanto, para el análisis entre el PBI y los indicadores macroeconómicos de cada país del G7 se decidió optar por el modelo de Mínimos Cuadrados Ordinarios (MCO), aprovechando la ventaja que permite una fácil interpretación y no es necesaria la conversión de variables. Este modelo está caracterizado por ser el mejor estimador linealmente insesgado, es decir, que arroja un estimador insesgado con varianza mínima, reduciendo al mínimo el error cuadrático del estimador.

\section{RESULTADOS}

Los datos provienen de información que elabora el FMI, la OCDE y el BCRP. De este modo, a partir de los datos obtenidos de las variables de interés, se estableció el impacto de la crisis de 2008 en la economía peruana y se realizaron las pruebas estadísticas (modelos regresivos) para establecer el impacto entre las variables de estudio.

En la figura 1 se observa que el Perú presentó un período de continuo crecimiento del PBI de $7,5 \%$ a $9,1 \%$ entre 
los años 2006 al 2008, respectivamente y finalizó en 2009, donde se experimentó el enfriamiento de la economía y el traslado a un equilibrio de menor crecimiento, obteniendo el 1,0\% del PBI, recuperándose los años siguientes.

En el caso de la economía de los países del G7, en el 2008 obtuvieron una ligera caída del 1,6\% (Canadá); 2,1\% (Francia); 2,5\% (Japón); 2,9\% (Reino Unido), 3,0\% (Estados Unidos) y 3,1\% (Alemania e Italia); de los cuales Japón, Reino Unido, Estados Unidos e Italia obtuvieron en el año 2008 un PBI negativo, en comparación con el 2006. Sin embargo, la crisis tuvo mayor impacto en el año siguiente, evidenciando un incremento en el déficit del PBI entre el 5,3\% y 9,4\%, obteniendo, los 7 países, un PBI negativo en el 2009. Reflejando una recuperación en su economía los años posteriores.

En cuanto al PBI per cápita, en la figura 2 se puede apreciar que las economías del G7 fueron afectadas por la crisis de forma negativa, pero teniendo distintos resultados tras su paso, con casos de recuperación del crecimiento como Alemania, Estados Unidos y Canadá obteniendo, entre los años 2009 al 2014, un incremento en su per cápita de 4
249,54; 3 421,62 y 3 150,19, respectivamente. Asimismo, se produjo un estancamiento en la economía, como es el caso de Francia que obtuvo un ligero incremento de 1 232,75 entre los años 2009 al 2014. También se evidencia una reducción de 1528,76 en la economía de Italia. En cuanto al Perú, hubo una ligera reducción en su per cápita en el año 2009, en comparación con el 2008; pero que fue recuperada y mantiene una trayectoria positiva, obteniendo un incremento en su per cápita de 2294 al 2014.

Con el objetivo de comprobar la hipótesis planteada, se realizó una prueba bajo el modelo ARIMA $(1,0,0)$ evaluando la ecuación de identidad del PBI (PBI=C+I+$\mathrm{G}+\mathrm{XN}$ ) y añadiendo la variable dicotómica crisis. De este modo, la variable endógena de un período t es explicada por las observaciones de ella misma correspondientes a períodos anteriores añadiéndose, como en los modelos estructurales, un término de error.

Tal como se observa en la tabla 1, el modelo presenta consistencia a nivel agregado y la variable crisis está relacionada negativamente con el PBI, alineándose con la hipótesis de que la crisis ha afectado la economía peruana, aunque

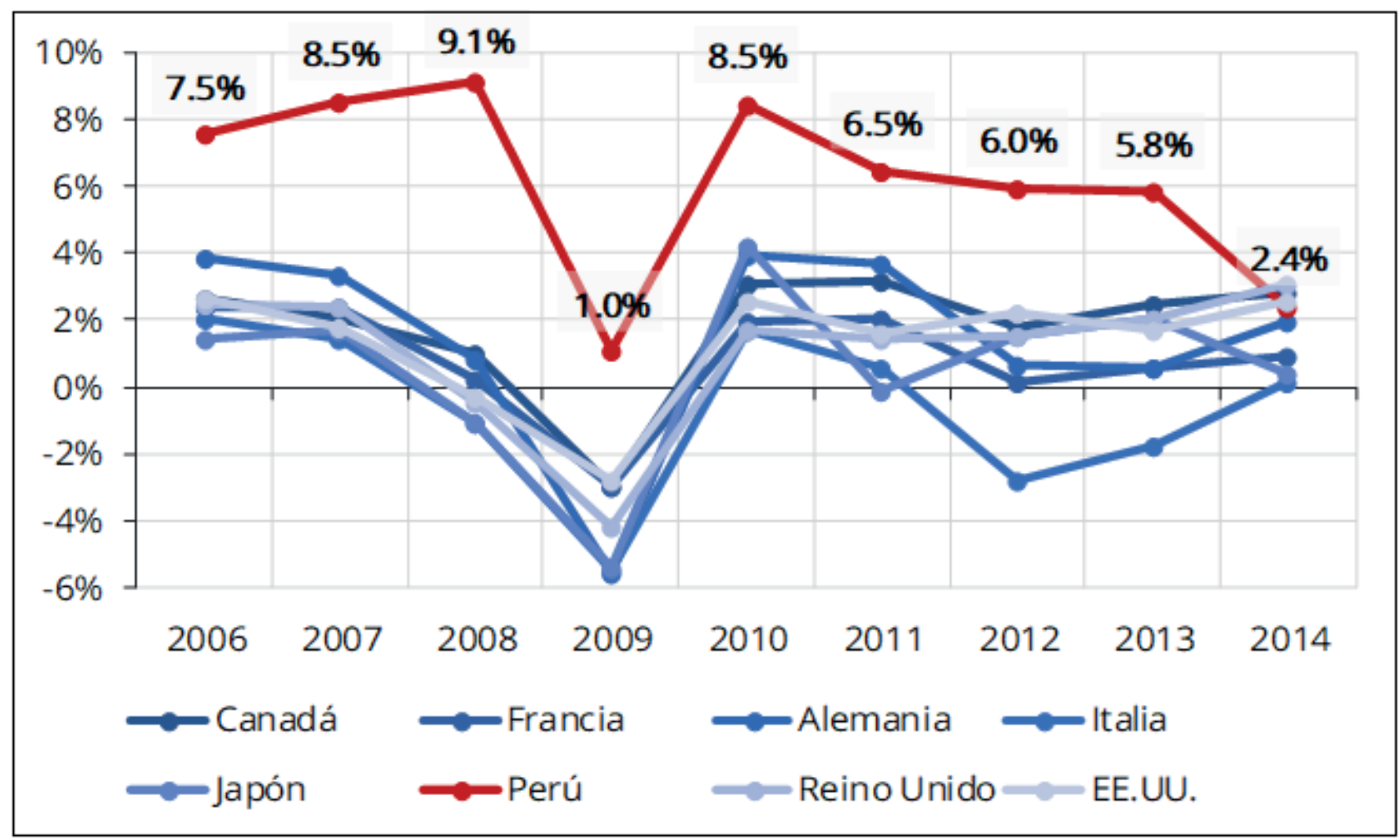

Figura 1. Crecimiento anual del PBI de Perú y países del G7 (Var. \% anual. Precios constantes) Fuente: Fondo Monetario Internacional (2018) / Elaboración propia. 


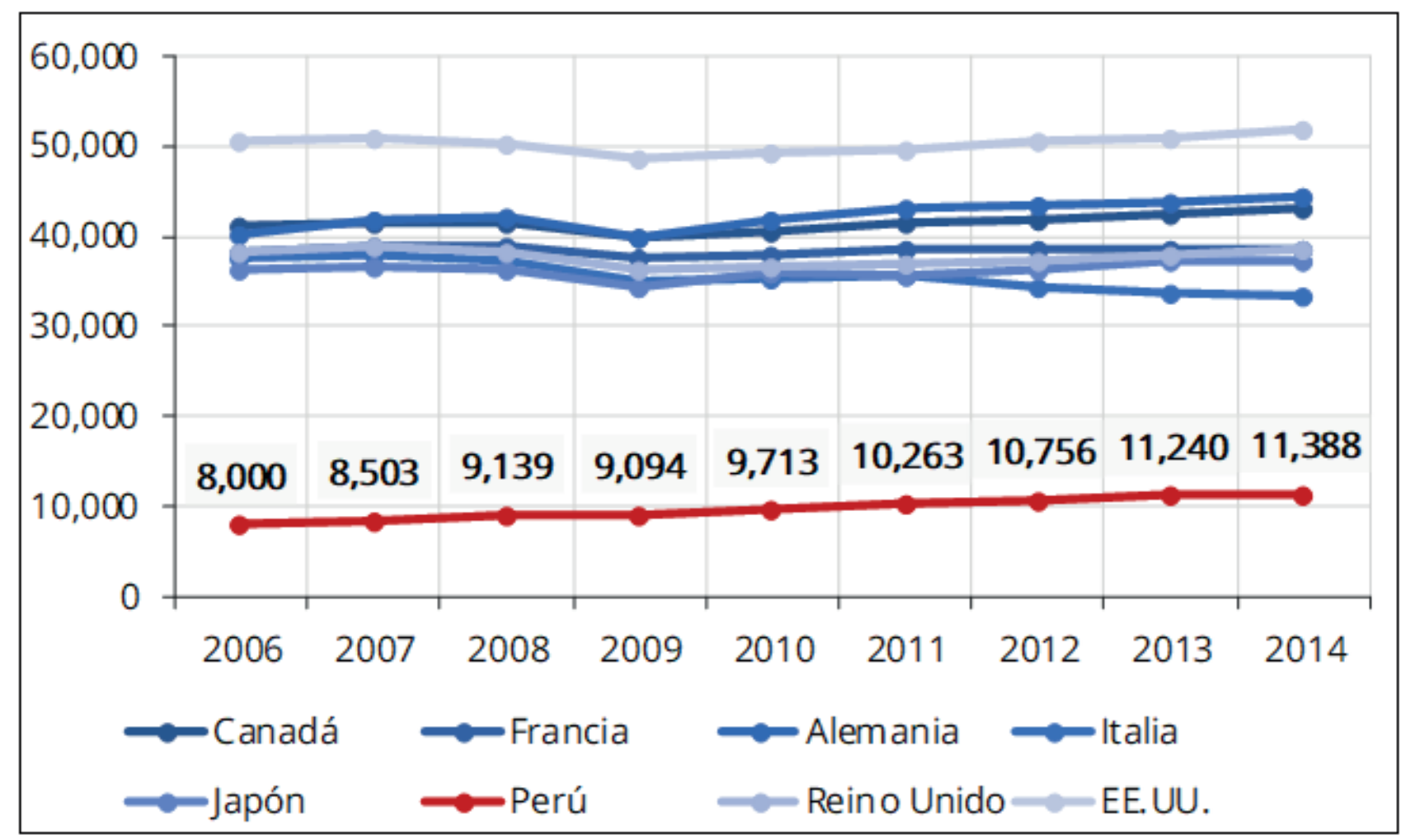

Figura 2. PBI per cápita: Perú y países del G-7 (US\$, PPP 2011)

Fuente: Fondo Monetario Internacional (2018) / Elaboración propia.

individualmente no presenta consistencia lo cual podría ser indicador que los efectos de la crisis no fueron directos ni inmediatos, comprobando de esta manera la hipótesis de estudio.

En efecto, la prueba ARIMA se realizó con un modelo autorregresivo de 1 período, utilizando la mayor cantidad de datos disponibles con frecuencia trimestral, por lo que se evaluó el período entre el primer trimestre de 1994 y el segundo trimestre de 2018, utilizando como variables las variaciones porcentuales de cada indicador y para la dicotómica el valor de 1 entre el primer trimestre de 2008 y el cuarto de 2014, y 0 para el resto de las observaciones.

Como se observa en la tabla 2, la correlación entre la variable dependiente y las independientes demuestran un alto grado de afinidad existente entre el PBI y consumo con un 0,6336 e inversión con un 0,7886 de relación positiva, reafirmando la teoría económica. Por otro lado, no hay una tendencia clara entre la variable dependiente y el gasto público, el cual está representado por un 0,2384 , denotando una reducida influencia posiblemente explicada por políticas públicas inefectivas en la producción nacional.
Los resultados de la tabla anterior se pueden observar en la figura 3, en la que se descarta que la relación entre el crecimiento del PBI durante el periodo 2008-2014 y la crisis tenga componente nulo, y en base al modelo empleado ARIMA se encuentra evidencia y certidumbre de una relación negativa entre el período investigado y la economía nacional peruana, de esta forma se está fortaleciendo nuestro supuesto estudiado. Sin embargo, también es pertinente recalcar que, al no existir una significancia individual, la correspondencia no ha sido preponderantemente directa o inmediata, sino que se presentó con impacto tardío en la economía del Perú.

\section{DISCUSIÓN}

El tema objeto de la presente investigación no tiene antecedentes principales o directos sobre las variables objeto de interés (crisis económica mundial de 2008 y Producto Bruto Interno peruano), ya que los estudios existentes abordan fundamentalmente el impacto de la crisis económica mundial de 1998 en el sistema financiero peruano. No obstante, a nivel nacional, De la Cruz (2010) realiza un estudio en el que argumenta que la crisis y desequilibrio del año 1998 representó un considerable impacto para el 
Tabla 1

Regresión ARIMA del PBI peruano (Las variables están expresadas en Var. \% anuales)

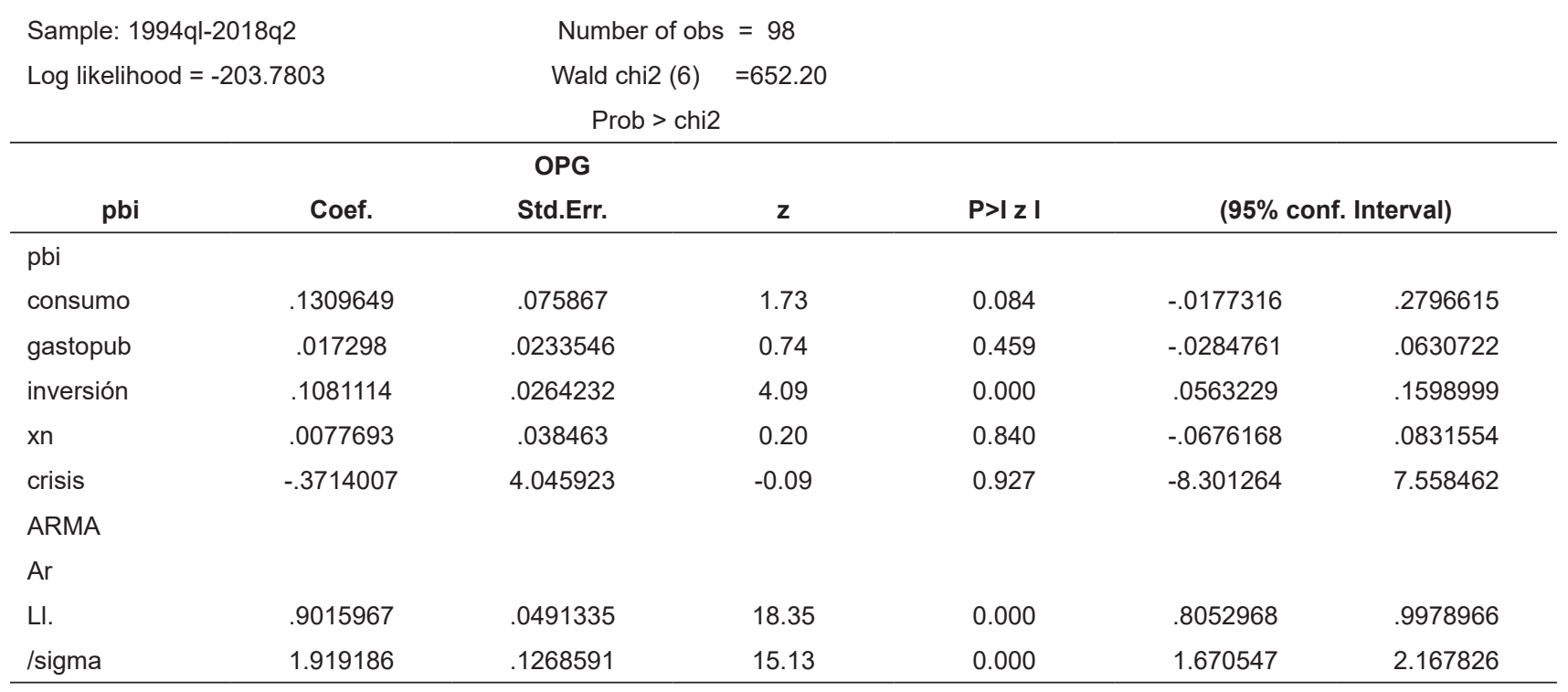

Fuente: Elaboración propia a partir de la aplicación del software Eviews 8.0.

Tabla 2

Regresión ARIMA de las variables de estudio en la correlación (Las variables están expresadas en \%)

\begin{tabular}{|c|c|c|c|c|c|c|}
\hline & pbi & consumo & gastopub & Invers $\sim n$ & $X n$ & crisis \\
\hline pbi & 1.0000 & & & & & \\
\hline consumo & 0.6336 & 1.0000 & & & & \\
\hline gastopub & 0.2384 & 0.0587 & 1.0000 & & & \\
\hline inversión & 0.7886 & 0.5964 & 0.3159 & 1.0000 & & \\
\hline$x n$ & 0.2725 & 0.2176 & 0.1146 & 0.3616 & 1.0000 & \\
\hline crisis & -0.1224 & -0.3371 & -0.1156 & -0.0970 & 0.1559 & 1.0000 \\
\hline
\end{tabular}

Fuente: Elaboración propia a partir de la aplicación del software Eviews 8.0.

sistema financiero del Perú. Considera que esta fase dejó importantes aspectos y lecciones que lo enriquecieron y que le permitieron enfrentar y resistir de mejor forma la flamante crisis financiera. A su vez, el fuerte impacto de la crisis y desequilibrio del sistema financiero del mundo han sido moderados o leves en el Perú, esto se debe a prácticas de una cabal disciplina fiscal y a la disposición adecuada de las finanzas públicas. Por su parte, a nivel internacional Ramírez (2009), si bien realiza un análisis descriptivo sobre las perturbaciones internacionales y fluctuaciones del Producto Bruto Interno, sus resultados solamente muestran una evidencia de República Dominicana. Sostiene que los factores o componentes externalizados desempeñan un punto muy importante en la determinación de las oscilaciones macroeconómicas cortoplacistas, en concreto en las fases que experimentó el Producto Bruto Interno. En ello, sopesó importantemente, el sector de bienes y activos, debido al impacto sobre las etapas de corto plazo de los shocks de brecha y desbalances del producto externo. La interpretación de impulso-respuesta (causa-efecto) cuantifica esta secuela más que proporcional en el largo plazo y al instante del shock de transición se da una respuesta en cierta medida rápida del nivel de actividad interno o doméstico. En estricto, la crisis económica como la crisis financiera de 2008, se ha producido principalmente debido al cambio de patrones de pensamiento. Se ha producido precisamente a causa de la fluctuación de confianza, envidia y resentimiento; de percepciones acerca de la naturaleza de la economía. En definitiva, los fenómenos y fluctuaciones económicas ocurridos a un nivel macroeconómico impactan a la economía mundial, y puesto que el Perú está inmerso en ella, siempre será influenciado y afectado por 


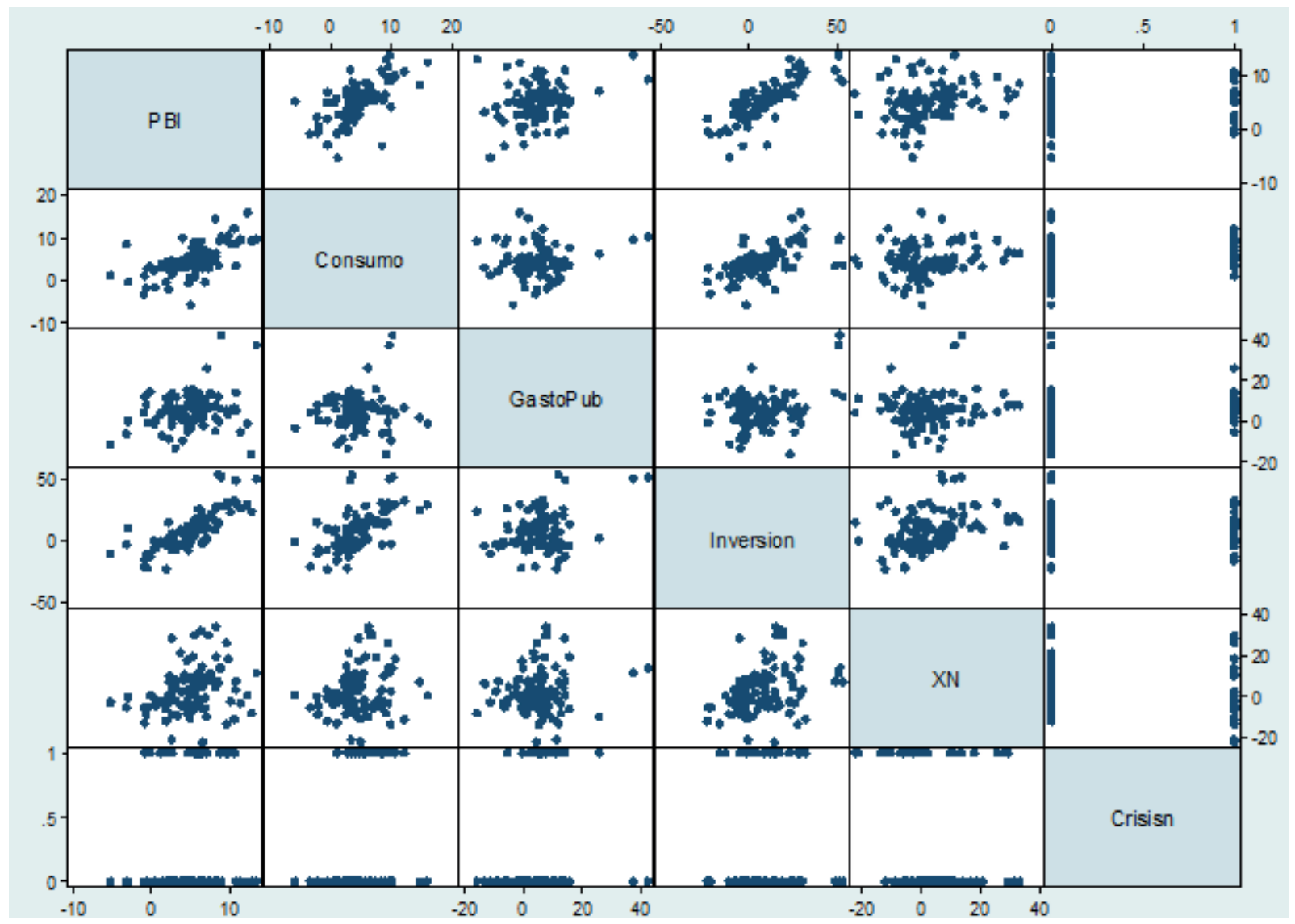

Figura 3. Correlación entre las variables de estudio

Fuente: Elaboración propia a partir de la aplicación del software Eviews 8.0.

los aspectos positivos (beneficios) o los aspectos negativos (perjuicios) que puedan acontecer. Empero, el nivel de incidencia o impacto dependerá de las actuaciones, tratados y convenios que pueda tener.

\section{REFERENCIAS}

Akerlof, G. y Shiller, R. (2014). Animal Spirits (3a ed.). España: Unión Editorial S.A.

Banco Central de Reserva del Perú. (2009). Fortalezas del Perú ante la crisis internacional. Revista Moneda, (139). Recuperado de: https://www.bcrp.gob.pe/publicaciones/revista-moneda/revista-moneda-139.html

Banco Central de Reserva del Perú. (2009). La crisis global 2007-2009 y la política monetaria del Banco Central de Reserva del Perú. Revista Moneda (139), 2326. Recuperado de: https://www.bcrp.gob.pe/docs/ Publicaciones/Revista-Moneda/Moneda-139/Moneda-139-04.pdf
De la Cruz, J. (2010). Impactos de la crisis económica mundial en el sistema financiero peruano y el desarrollo de un sistema de prevención de fragilidad financiera, a través de un modelo econométrico (Tesis para optar el título de economista). Universidad de Piura.

Fondo Monetario Internacional. (2014). Monetary and Macroprudential Policies to Manage Capital Flows. International Monetary Found. Recuperado de: https:// www.imf.org/en/Publications/WP/Issues/2016/12/31/ Monetary-and-Macroprudential-Policies-to-Manage-Capital-Flows-41347

Herrera, B. (2003). Globalización y sistema financiero. Lima: Fondo Editorial Universidad Nacional Mayor de San Marcos.

Krugman, P. (2009). De vuelta a la economía de la gran depresión y la crisis de 2008. Colombia: Grupo Editorial Norma.

Mankiw, G. (2014). Macroeconomía (8a ed.). España: Antoni Bosch editor, S.A. 
Organización para la Cooperación y el Desarrollo Económicos. (2016). Caminos de Desarrollo. Estudio multidimensional del Perú Volumen 2. Análisis detallado y recomendaciones. Recuperado de: http://www. oecd.org/dev/americas/RESUMEN_EJECUTIVO_ MDCR_Peru.pdf

Ramírez, F. (2009). Perturbaciones Internacionales y Fluctuaciones del Producto Interno Bruto en una Economía en Desarrollo: Evidencia de República Dominicana para el Período 1998-2008 (Tesis para optar el grado académico de magister en economía). Pontificia Universidad Católica de Chile.
Stiglitz, J. (2013). El informe Stiglitz. La reforma del sistema económico en el marco de la crisis global. México: RBA Libros S.A.

Sunstein, C - Thaler, R. (2017). Un pequeño empujón. España: Editorial Taurus. 